\title{
A proposal for testing kit of corona viruses using 3D photonic structure
}

\author{
Sangram Kishore Mohanty ${ }^{1} \cdot{\text { Subhankar } \text { Das }^{2} \cdot \text { K. P. Swain }}^{3} \cdot$ Urmila Bhanja $^{4} \cdot$ G. Palai $^{3}$ (I)
}

Received: 28 August 2020 / Accepted: 26 September 2020/Published online: 29 October 2020

(C) Springer-Verlag GmbH Germany, part of Springer Nature 2020

\begin{abstract}
Design of corona virus testing kit is proposed in this paper using silicon based 3D photonic structure through zirconium quantum dot solution at the signal of $412 \mathrm{~nm}$. The principle of measurement depends on the computation of reflectance, absorbance and transmittance of virus based quantum dot solution. In this paper, the reflectance is studied through the analysis of photonic band gap and absorbance is made through its numerical treatment. Further, the numerical investigation shows that the transmitted energy through photonic structure would determine the type of corona virus. For example; if the transmitted energy lies within the visible spectrum the sample would be normal corona virus. However, the sample could be IBV (SARS COV-2) if the transmitted energy would be Infrared.
\end{abstract}

\section{Introduction}

A novel corona virus is a new damage that has not been previously identified in humans. However, it is originated in the Wuhan city of China in December 2019. The disease infects the people and the rate of infection grows exponentially among the people. The corona virus has large family of viruses, which cause the illness ranging from severe diseases (MERS and SARS) (Cui et al. 2019; Forni et al. 2017). However, all common colds do not belong to the novel corona viruses. There are different corona viruses such as N5H1, N5H2, H9N2, H4N6, FAdV, and IBV (Ahmed et al. 2018). The infectious bronchitis viruses (IBV) have different characteristics with respect to the others. Moreover, the properties of the IBV are similar to the present novel corona viruses (Crossley et al. 2012; Editorial Commentary 2020). So, SARS COV-2 or novel corona virus or COVID-19 belongs to the family of IBV. It is caused by sever acute respiratory syndrome. Basically, such viruses spread through the close contact via talking,

G. Palai

gpalai28@gmail.com

1 Bijupatnaik University of technology, Rourkela, Odisha, India

2 Duy Tan University, Da Nang, Vietnam

3 Department of Electronics and Communication Engineering, Gandhi Institute for Technological Advancement, Bhubaneswar, India

4 Indira Gandhi Institute of Technology, Sarang, Odisha, India coughing, sneezing and through the touching of contaminated surface. Moreover, the common symptoms of these diseases are fever, tiredness, breathing problem, and the loss of smell aside cough and sneeze. The time of exposure of such diseases is about 5-6 days. However, currently the time range of 2 to 14 days are considered as the time of exposure. However, it is extended to 21 days in some cases. Since medicines are not available for such disease, hydrochloride based medicine and plasma therapy are applied on a hit and trial basis. However, it is important to increase the immune system of the patients to keep away such diseases. Though no real medicine have been used for the same so far, some preventive measures including covering mouth while coughing and sneezing, frequently hand washing with soap, wearing a face mask in public places, maintaining distances and self isolation for minimum 14 days, have been advised and recommended now a days. As far as diagnosis of this disease is concerned, reverse transcription polymerase chain reaction (RTPCR) of infected secretion or CT imaging of the chest are used to know the status of the disease (CT provides best diagnosis for COVID-19 2020; Ai et al. 2020). Ribonucleic acid (RNA) testing of respiratory is used as standard test for SARA CoV-2. Recently various laboratories and companies are designing serolaboratories (Coronavirus disease (COVID-19) technical guidance: Laboratory testing for 2019-nCoV in humans, World Health Organization 2020), which will diagnose the antibody of the same. However the accuracy is the matter of concerned here. Imaging features of the chest radiograph and computer tomography are used for symptomatic nature (Vogel (2020). Further comparing 


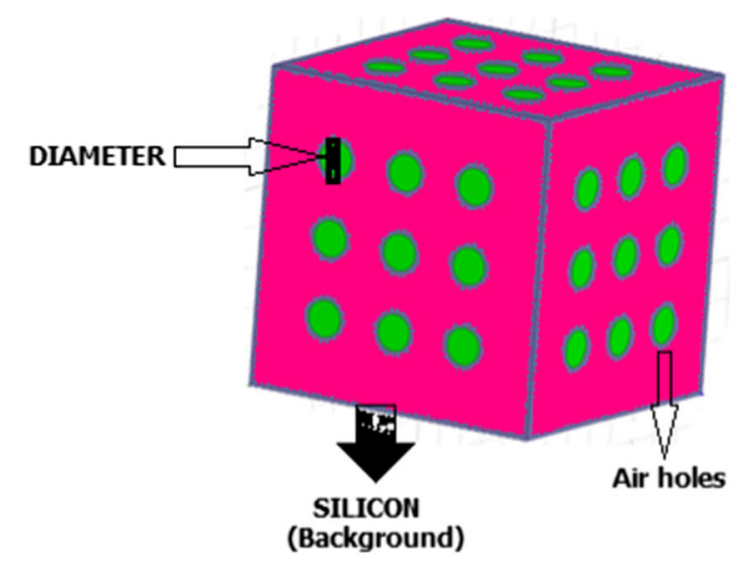

(a) Schematic diagram of 3D photonic structure

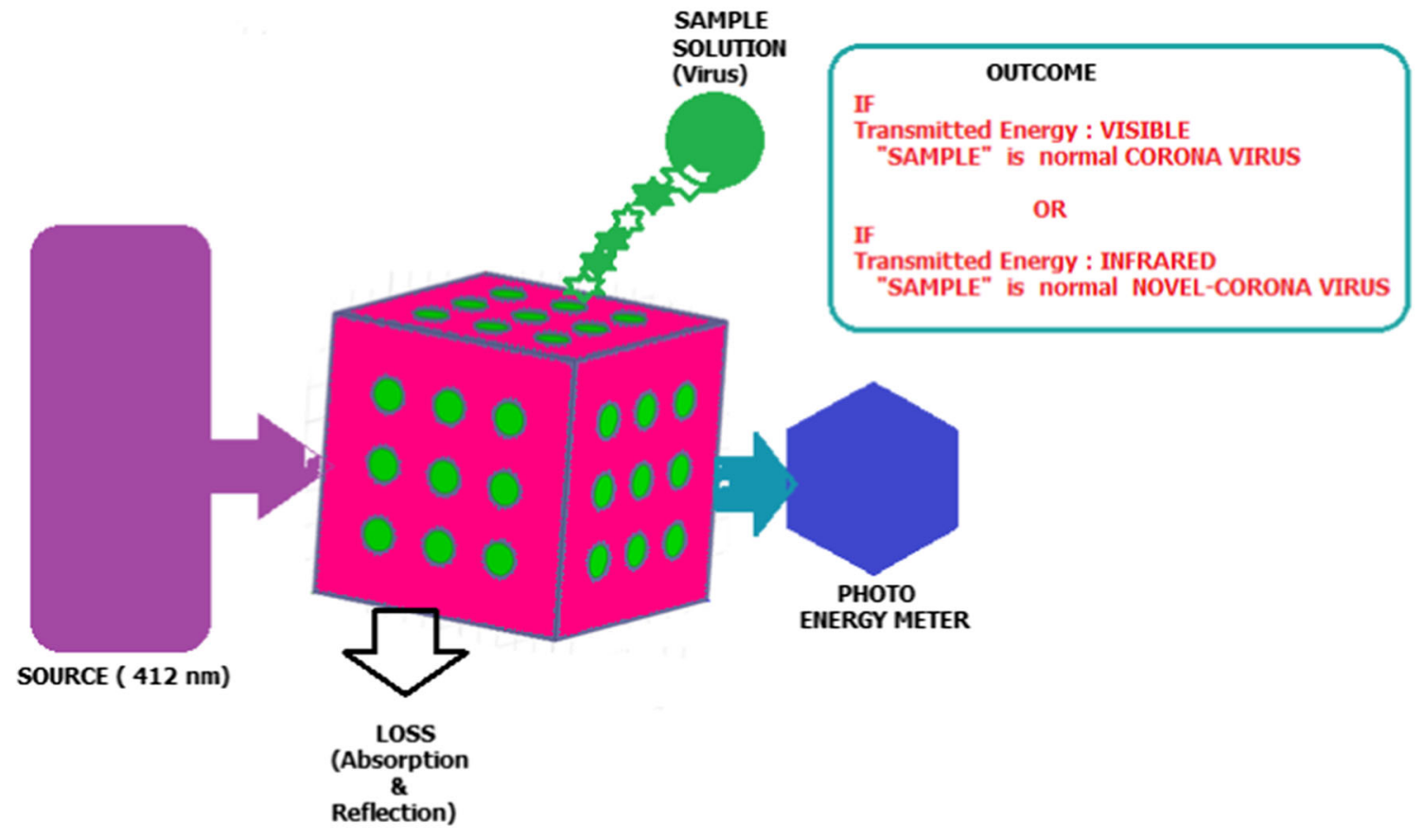

(b) Operational mechanism of investigation of investigation of corona virus

Fig. 1 a Schematic diagram of 3D photonic structure. b Operational mechanism of investigation of investigation of corona virus

these two tests, computed tomography is less specific with respect to PCR but it is faster and more sensitive (McNamara 2020). Though different methods are available to investigate and detect the presence of corona virus. The proposed technique distinguishes the novel corona from normal corona easily, which is realised through the zirconium quantum dot solution via 3D photonic structure. The preparation of zirconium quantum dot solution is indicated in the reference (Ahmed et al. 2018) where photo luminescence has been studied. Extrapolating requisite data from the reference Ahmed et al. (2018) and applying the numerical approach, a method is proposed in the current paper to identify the sample whether it is affected by novel corona or not.

\section{Structure and mechanism}

The aim of the proposed work is to identify novel corona virus from different corona viruses. To do so, zirconium quantum dot solution is mingled with the sample. This solution is infiltrated in the proposed $3 \mathrm{D}$ structure which is made up silicon as background material and $3 \times 3$ air holes have been etched, which is shown in the Fig. 1a.

Figure 1a represents schematic diagram of 3D photonic structure where diameter of air hole and lattice spacing is shown. Though the proposed structure is not new pertaining to the measurement of bio-specimen (Palai and Tripathy (2014); Palai and Dhir 2015; Swain and Palai 2019), the operational mechanism is different from other works. 


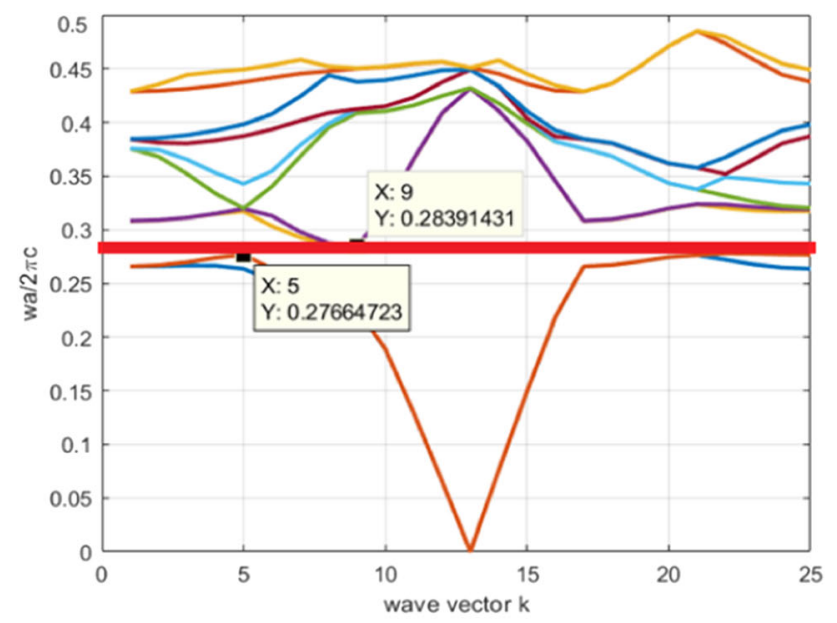

(a) Photonic band gap structure of 3DPC for corona virus N5H1

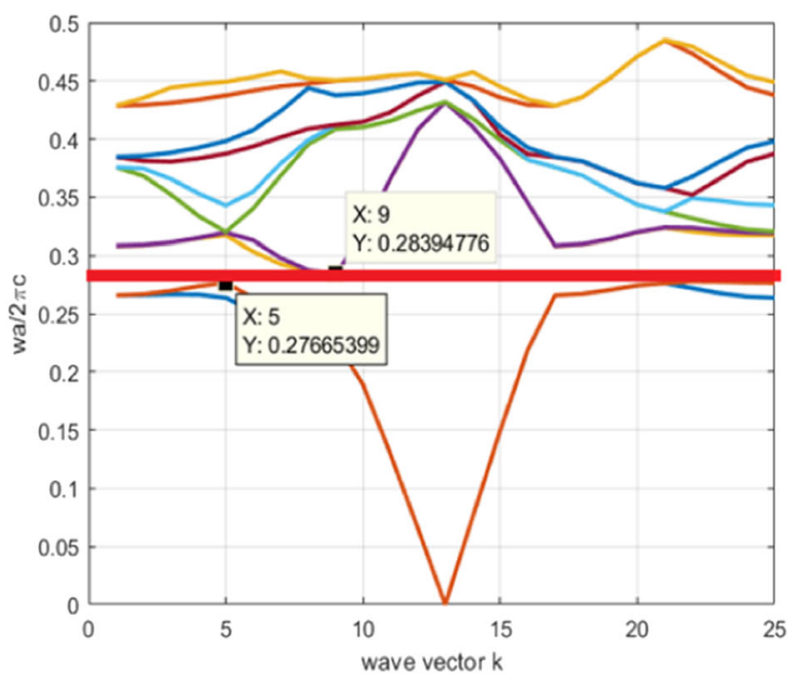

(b) Photonic band gap structure of 3DPC for corona virus N5H2

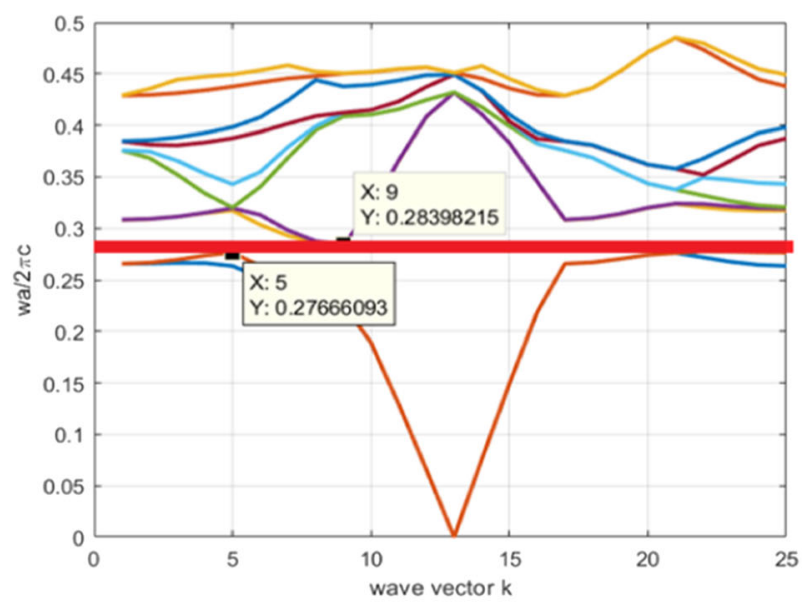

(c) Photonic band gap structure of 3DPC for corona virus H9N2
4Fig. 2 a Photonic band gap structure of 3DPC for corona virus N5H1. b Photonic band gap structure of 3DPC for corona virus $\mathrm{N} 5 \mathrm{H} 2$. C Photonic band gap structure of 3DPC for corona virus H9N2. d Photonic band gap structure of 3DPC for corona virus H4N6. e Photonic band gap structure of 3DPC for corona virus FAdV. f Photonic band gap structure of 3DPC for corona virus IBV

The same working mechanism can be understood from the Fig. 1b.

Figure $1 \mathrm{~b}$ represents an operational mechanism to investigate the status of corona virus through the realisation of output energy. Here, the signal of $412 \mathrm{~nm}$ originating from the source is incident on the photonic structure where the corona virus solution has been infiltrated in the air holes. The incident signal interacts with the structure and suffers from absorption as well as reflection loss. Finally, the transmitted energy would be appeared in the photo energy meter at the output end. Basically, the amount of transmitted energy is a function of both absorbance and reflectance. In this case the reflection loss is computed through the photonic band gap analysis, where the absorbance is computed through the analytical treatment. Further, the computational result of both reflectance and absorbance relies on the intrinsic parameters (Refractive indices of background material, infiltrated corona viruses, diameter of air holes, lattice spacing of the structure, configuration of the structure and nature of the material) of the proposed structure at the signal of $412 \mathrm{~nm}$. To realise the same investigation, the lattice spacing and diameter of air holes are considered to be of $1000 \mathrm{~nm}$ and $880 \mathrm{~nm}$ respectively. Finally, the output energy determines the specimen affected by IBV (novel corona virus) or other viruses such as N5H1, N5H2, H9N2, H4N6, FAdV. For example; if transmitted energy lies within infrared regime then the specimen belongs to SARS COV 2. Similarly, the specimen belongs to other category if output signal lies within the visible regime.

\section{Mathematical treatment}

Primarily, the investigation of the nature of corona viruses depends on the output energy which is transmitted through the photonic structure. From section 2, it is realised that such transmitted energy is a function of absorbance as well as reflectance. The absorbance is computed using the analysis of PL intensities via $\mathrm{Zr}$ QD solutions where the reflectance is obtained through the analysis of photonic band gap of proposed 3D structure with the help of plane wave expansion technique (Dhasarathan et al. 2020).

The basic equation for absorbance can be written as 


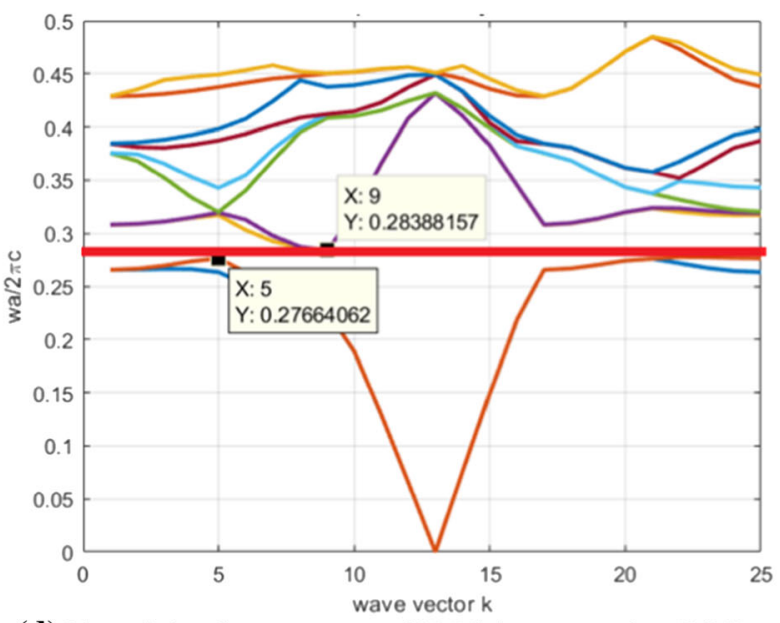

(d) Photonic band gap structure of 3DPC for corona virus H4N6

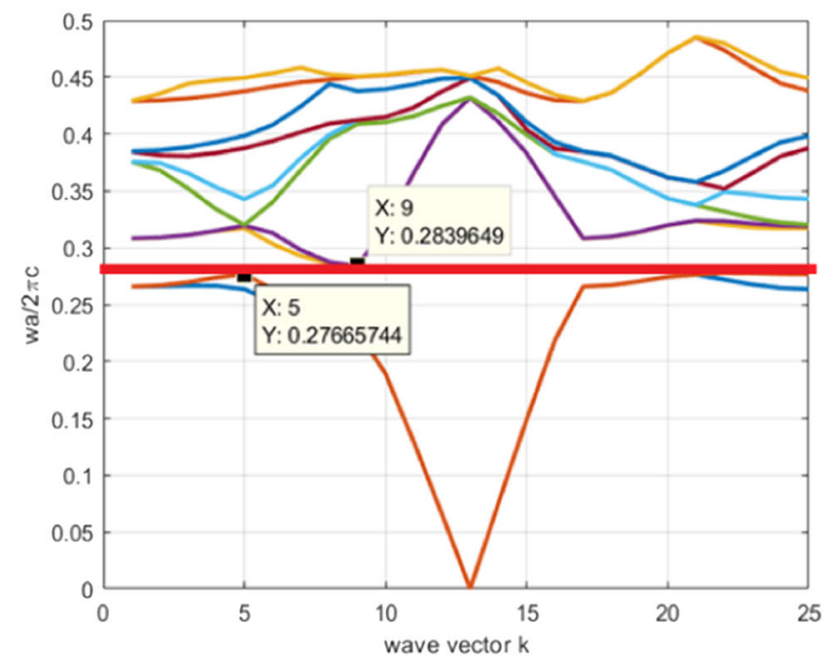

(e) Photonic band gap structure of 3DPC for corona virus FAdV

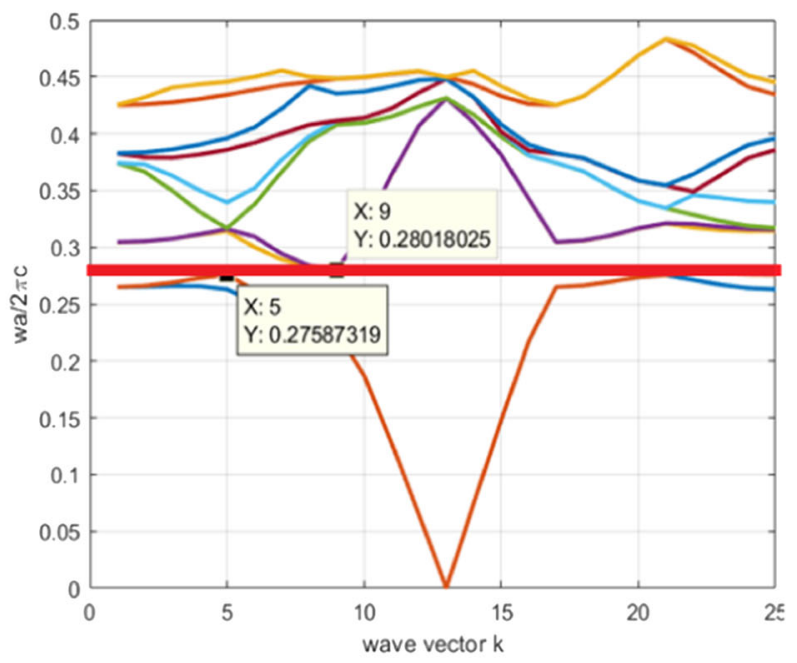

(f) Photonic band gap structure of 3DPC for corona virus IBV

Fig. 2 continued

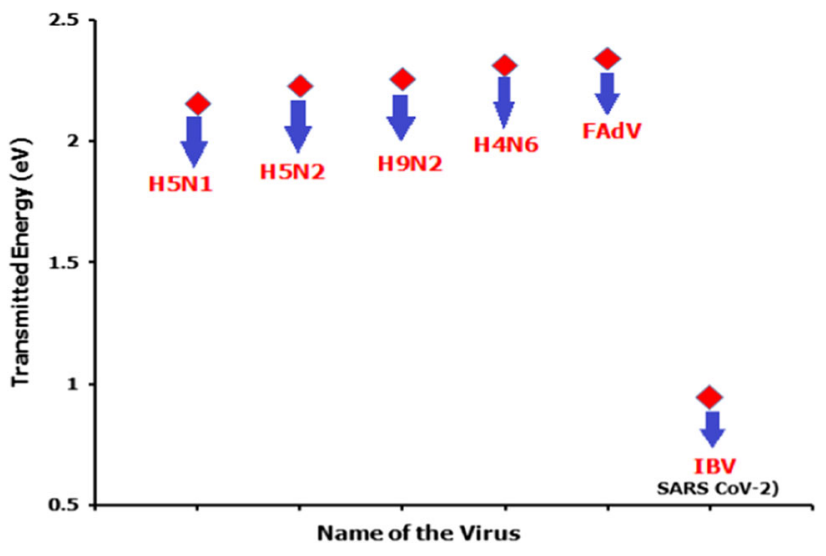

Fig. 3 Transmitted energy of different corona viruses

$A=e^{-(\beta d+\alpha T)}$

where, $\beta$ and $\mathrm{d}$ be the absorption coefficient (Schubert 2004) and thickness of silicon respectively. $\alpha$ and T be the absorption coefficient and thickness of the structure respectively. Furthermore, the absorption coefficient can be written in terms of photo tumescence as Tomar et al. (2012)

$\alpha=\frac{1}{T} \log _{e}(P L)$

Similarly, the reflectance can be determined with the help of photonic band gap of proposed silicon based three dimensional photonic crystal structure. The amount of energy lies within the photonic band gap equals to the energy is reflected by the structure (Dhasarathan et al. 2020).

Finally the transmitted energy $\left(\mathrm{E}_{\mathrm{t}}\right)$ is written in terms of initial energy $\left(\mathrm{E}_{\mathrm{i}}\right)$ as

$E_{t}=T \times E_{i}$

where, $\quad T=1-A-R \quad$ (A-absorbance and $\mathrm{R}-$ reflectance)

\section{Result and discussion}

From Sections 2 and 3, it is realised that the transmitted energy is a basic parameter to identify the nature of the corona viruses. Further, it is also observed that the transmitted energy is a function of both reflectance and absorbance including the input energy. The reflectance is computed through the analysis of photonic band, which is made with the help of plane wave expansion method. Moreover, the photonic band gap is a function of refractive indices, lattice spacing $(1000 \mathrm{~nm})$ and radius of air holes $(880 \mathrm{~nm})$. The refractive indices can be obtained from the reference Ahmed et al. (2018) after analysing PL of 
different corona viruses. Keeping these values in the mind, the result for photonic band of the 3D structure is shown in the Fig. 2a-f for different viruses such as N5H1, N5H2, H9N2, H4N6, FAdVand IBV (SARS CoV-2) respectively.

In Fig. 2; the normalised frequency $(\mathrm{a} / \lambda)$ and wave vector $(\mathrm{k})$ is taken along vertical and horizontal axis respectively. In these figures different allowed and disallowed bands are found, where photonic band gap is shown in green and red colour. Moreover, the lower and higher values of the normalised frequency are inset in all diagrams of Fig. 2. Further, the wavelength corresponding to the upper and lower normalised frequencies are obtained. Again, subsequently the amount of energy corresponding to each band can be computed, which is represented as the amount of energy reflected by the structure. After determining the reflected energy, the reflectance (reflected energy /incident energy) is computed. Furthermore, the absorbance is computed using Eqs. (1) and (2), where the absorption coefficient is computed through PL intensities of different viruses. Finally, the absorbance corresponding to each virus is obtained. With the help of Eq. (3), the transmittance and consequently the output energy is also computed. The variation of different output energy with respect to different viruses is shown in the Fig. 3.

In Fig. 3; the transmitted energy in $\mathrm{eV}$ is taken along vertical and name of virus is taken along horizontal axis respectively. After analysing, the Fig. 3, it is found an interesting result pertaining to the transmitted energy. For example the photon energy varies from 2.25 to $2.34 \mathrm{eV}$. $(2.15193 \mathrm{eV}, 2.229034 \mathrm{eV}, 2.256074 \mathrm{eV}, 2.312416 \mathrm{eV}$, and $2.339587 \mathrm{eV}$ for N5H1, N5H2, H9N2, H4N6, and FAdV respectively) for all the viruses except IBV, whose value is found to be $0.942969 \mathrm{eV}$. Again analysing the aforementioned results, it is found that the output energy for viruses $\mathrm{N} 5 \mathrm{H} 1, \mathrm{~N} 5 \mathrm{H} 2, \mathrm{H} 9 \mathrm{~N} 2, \mathrm{H} 4 \mathrm{~N} 6$, FAdVlies within the range of visible spectrum, where the energy lies within the IR spectrum corresponding to the SARS CoV-2 (IBV). So the outcomes of the investigation indicates that transmitted signal decides whether the sample is affected with novel corona virus or not.

\section{Conclusion}

A proposal for testing the presence of corona viruses is made with the help of 3D photonic crystal structure. The principle of mechanism deals with the transmittance, reflectance and absorbance at the signal of $412 \mathrm{~nm}$. Photonic band gap analysis and analytical treatment computes the outcomes of reflectance and absorbance respectively. The final result indicates that the sample may be affected by SARS CoV-2 if the transmitted energy would be in the
IR regime. Similarly, it will be affected by usual corona viruses if output energy lies within visible regime.

\section{References}

Ahmed SR, Kang SW, Oh S, Lee J, Neethirajan S (2018) Chiral zirconium, quantum dots: a new class of nanocrystals for optical detection of coronavirus. Heliyon 4:Nowe00766

Ai T, Yang Z, Hou H, Zhan C, Chen C, Lv W, Tao Q, Xia L (2020) Correlation of chest $\mathrm{CT}$ and RT-PCR testing in coronavirus disease 2019 (COVID-19) in China: a report of 1014 cases. Radiology. https://doi.org/10.1148/radiol.2020200642.PMID32101510

Coronavirus disease (COVID-19) technical guidance: Laboratory testing for 2019-nCoV in humans,WorldHealth Organization (2020) Archived from the original on 15 March 2020. Retrieved 14 March 2020. https://apps.who.int/iris/bitstream/handle/10665/ 331329/WHO-COVID-19-laboratory-2020.4-eng.pdf

Crossley BM, Mock RE, Callison SA, Hietala SK (2012) Identification and characterization of a novel alpaca respiratory coronavirus most closely related to the human coronavirus $229 \mathrm{E}$. Viruses 4:3689-3700

CT provides best diagnosis for COVID-19 (2020) ScienceDaily, Retrieved 14 March 2020. https://www.sciencedaily.com/ releases/2020/02/200226151951.htm

Cui J, Li F, Shi ZL (2019) Origin and evolution of pathogenic coronaviruses. Nat Rev Microbiol 17:181-192

Dhasarathan V, Sahu SK, Nguyen TK, Palai G (2020) Realization of all logic gates using metamaterials based three dimensional photonics structures: a future application of 3D photonics to optical computing. Optik 202:163723

Editorial Commentary (2020) One Health. One Health 9:100123

Forni D, Cagliani R, Clerici M, Sironi M (2017) Molecular evolution of human coronavirus genomes. Trends Microbiol 25:35-48

McNamara A (2020) Coronavirus antibody tests: how they work and when we'll have them, BBC Science Focus Magazine, Retrieved 6 April 2020. https://www.sciencefocus.com/news/coronavirusantibody-tests-how-they-work-and-when-well-have-them/

Palai G, Tripathy SK (2014) Measurement of glycerol concentration in B-H-G solution using 3D photonic crystal structure. Optik Int J Light Electron Opt 125:2875-2879

Palai G, Dhir TK (2015) Theoretical model to measure the concentration of haemoglobin in human blood using 3D photonic crystal structure. Optik 126:478-482

Schubert EF (2004) Materials-refractive-index-and-extinction-coefficient, 2004, https://www.rpi.edu/schubert/Educational-resources/ Materials-Refractiveindex-and-extinction-coefficient.pdf (28.03.11)

Swain KP, Palai G (2019) Measurement of haemoglobin of human blood using 3-D photonic structure via machine learning J.K. Moharana. Optik Int J Light Electron Opt 179:582-586

Tomar AK, Mahendia A, Chahal RP, Kumar S (2012) Photoluminescence and refractive index behaviour of PMMA-PAni blends. In: AIP Conf. Proc., p 1447

Vogel G (2020) New blood tests for antibodies could show true scale of coronavirus pandemic, Science Magazine. Retrieved 6 April 2020.https://www.sciencemag.org/news/2020/03/new-bloodtests-antibodies-could-show-true-scale-coronavirus-pandemic

Publisher's Note Springer Nature remains neutral with regard to jurisdictional claims in published maps and institutional affiliations. 